\title{
SOME REMARKS ON THE STABILITY OF TTME-VARYING SYSTEMS
}

by

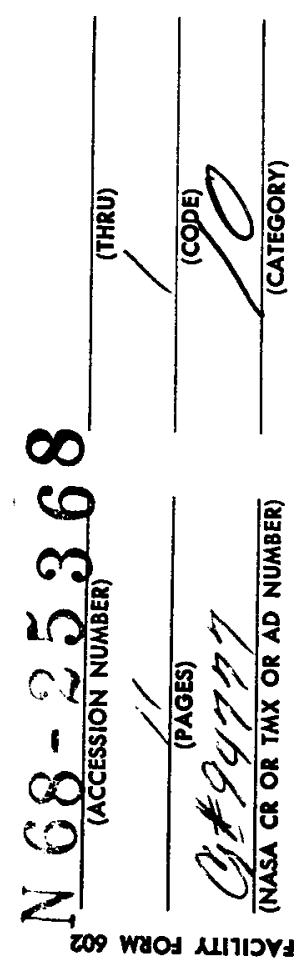

\author{
J. K. Aggarwal* \\ Department of Electrical Engineering \\ The University of Texas \\ Austin, Texas
}

and

E. F. Infante $e^{* *}$

Division of Applied Mathematics

Brown University

Providence, Rhode Island
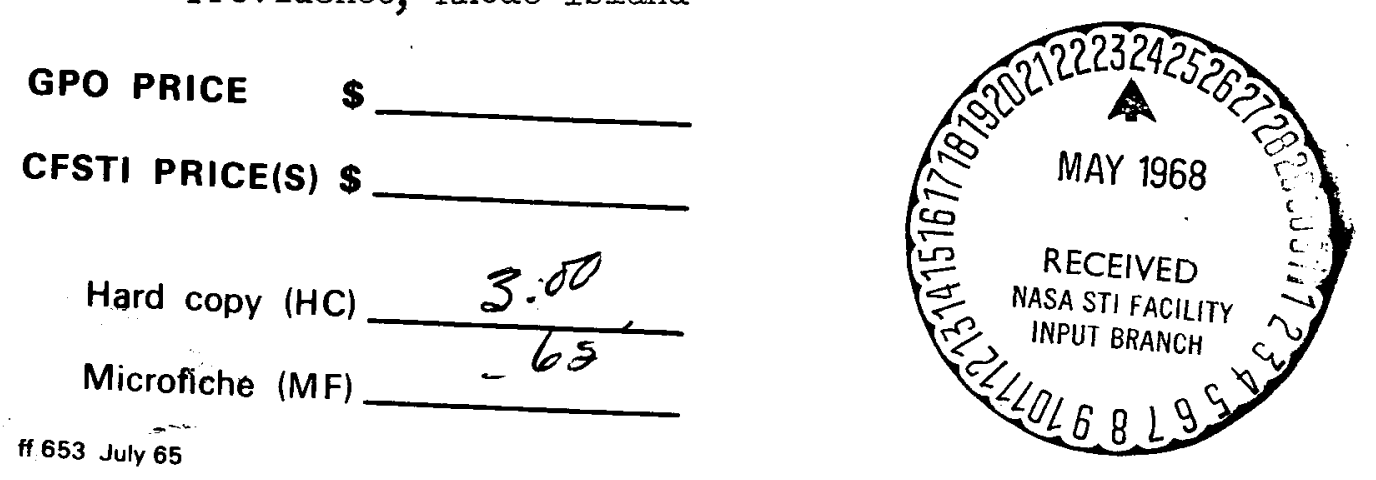

* This research was supported by the National Science Foundation under Grant GK-1879.

** This research was supported in part by the Air Force Office of Scientific Research, Office of Aerospace Research, United States Air Force, under AFOSR-693-67, in part by the National Aeronautics and Space Administration under Grant 170, 40-002-015 and in part by the United States Army under Grant No. DA-31-124-ARO-D-270. 
SOME REMARKS ON THE STABILITY OF TTME-VARYING SYSTEMS

A large number of results are presently available for the determination of stability conditions for linear time-varying systems; among these is the well known "circle criterion" [1] and its variants. The object of this note is to aiscuss through exampies some of the results which are of an essentially different nature from the circle criterion. The examples chosen are of second order but they are rather illustrative of some of the advantages and difficulties of the techniques discussed.

Example 1: Consider the system, first discussed by Marcus and Yamabe [2]

$$
\dot{x}=A x ; A(t)=\left[\begin{array}{ll}
-1+a \cos ^{2} t & 1-a \sin t \cos t \\
-1-a \sin t \cos t & -1+a \sin ^{2} t
\end{array}\right] \text {, }
$$

with $a>0$. The state transition matrix for this system is

$$
x(t)=\left[\begin{array}{cc}
e^{(a-1) t} \cos t & e^{-t} \sin t \\
-e^{(a-1) t} \sin t & e^{-t} \cos t
\end{array}\right] \text {, }
$$

which shows that for asyiuptotic stability ore musi have a<i. The 
eigenvalues of the system are given by

$$
\lambda_{1,2}=\frac{a-2 \pm \sqrt{a^{2}-4}}{2}
$$

and are time invariant; they lie in the left-hand plane for $a<2$.

Example 2: The second order equation

$$
\ddot{x}+2 \xi \dot{x}+[1+f(t)] x=0
$$

with $\xi>0,|f(t)|<\infty$, and $E\{f(t)\}=\lim _{t \rightarrow \infty} \frac{1}{t-t_{0}} \int_{0}^{t} f(\tau) d \tau=0$ is a simple gneralization of the damped Mathieu equation.

Example 3: Consider the simple modification of the above equation

$$
\ddot{x}+2 g(t) \dot{x}+f(t) x=0
$$

with $0<\underline{g} \leqq g(t) \leqq \bar{g}$ and $0<\alpha \leqq f(t)-2 \dot{g}(t) \leqq \beta$.

These three examples constitute the nucleus of the following discussion. 
Stability Results

There seem to be two possible viewpoints for the determination of stability conditions for time-varying systems. One of these viewpoints is to insist that the "frozen" system be stable for a range of variation of the time-varying parameters and then to further restrict this range and perhaps impose bounds on the rate of change of these parameters to obtain sufficient conditions for stability. This is the viewpoint which leads to the circle criterion. It is self-evident that such a viewpoint can be, in some cases, extremely restrictive. Stability is a concept which demands observation for all subsequent times after the starting time, and it is not difficult to conceive of a system which is alternately frozen time stable and frozen time unstable but which is asymptotically stable in the sense of Liapunov.

The second possible viewpoint is to consider the time-varying system as a perturbation on an "average" autonomous system. If this average system is asymptotically stable then for sufficiently small perturbations, in some appropriate sense, it is to be expected that the time-varying system will be stable. A stability result of this type in which the averaging is done over the time has been obtained in [3]:

Theorem: The time-varying system $\dot{\mathrm{x}}=\mathrm{A}(\mathrm{t}) \mathrm{x}, \mathrm{A}(\mathrm{t})$ bounded, is almost surely asymptotically stable if, for some positive definite matrix $B$ anā some $\epsilon>\hat{0}$ 


$$
\lim _{t \rightarrow \infty} \frac{1}{t-t_{0}} \int_{t_{0}}^{t} \lambda_{\max }\left[A^{\prime}(\tau)+B A(\tau) B^{-1}\right] d \cdot \tau \leqq-\epsilon .
$$

Furthermore, for sufficiently large times

$$
x^{\prime}(t) B x(t) \leqq x^{\prime}\left(t_{0}\right) B x\left(t_{0}\right) e^{-\epsilon\left(t-t_{0}\right)}
$$

This theorem represents an attempt to draw information from the behavior of the eigenvalues of $A(t)$. Example 1 shows that the eigenvalues of $A(t)$ do not carry a great deal of information; on the other hand, the above theorem indicates that the "average" in the time sense of the eigenvalues of $A^{\prime}(t)+B A(t) B^{-1}$ do. Naturally, the above theorem only gives sufficient conditions and is not constructive in the sense that nothing is said about matrix $B$. It should be noted that this result includes the familiar stability criterion that the eigenvalues of. $A^{\prime}(t)+A(t)$ be negative.

This simple theorem, in the case of Example 1, yields the best possible result by letting the matrix $B$ be the identity matrix; the number $\epsilon$ can then be taken as $2-2 a$ and then it becomes necessary for stability that $a<1$. From the transition matrix it is clear that

$$
x^{\prime}(t) x(t)=x^{\prime}(0)\left[\begin{array}{ll}
e^{2(a-1)} t & 0 \\
0 & e^{-2 t}
\end{array}\right] x(0)
$$


while the theorem yields $x^{\prime}(t) x(t) \leqq x^{\prime}(0) x(0) e^{2(a-1) t}$ for sufficiently large times.

Perhaps of more interest is the application of this theorem to Example 2 in standard phase space. In this case letting

$$
B=\left[\begin{array}{ll}
1+2 \xi^{2} & 3 \\
\xi & 1
\end{array}\right],
$$

an elementary computation yields that $\epsilon$ can be taken as

$$
\epsilon=2 \xi-\left(\frac{4 \xi^{4}}{1+\xi^{2}}+\frac{E\left\{f^{2}\right\}}{1+\xi^{2}}\right)^{\frac{1}{2}}
$$

which yields as a sufficient condition for stability that $E\left\{f^{2}\right\}<$ $4 \xi^{2}$. It is interesting to note, in this case, that among the functions $f(t)$ for which the system is asymptotically stable is an odd periodic square wave of height $2 \xi-\delta$, for some small positive $\delta$. However, for $\xi>\frac{1}{2}$ and such a wave the system is not frozen time stable when $f(t)=-2 \xi+\delta$.

It is also of interest to compare this result to two recent ones. Michael [4] has considered by the Liapunov method the equation

$$
\ddot{x}+\frac{1}{2} \delta \dot{x}+\left(\omega^{2}+\mu \cos 2 t\right) x=0
$$


and in this notation, after an appropriate time scaling, the above result yields as a sufficient condition for asymptotic stability that

$$
\mu<\frac{\wp \omega}{2}
$$

rather than the much more meager and more complicated result obtained in [4]. The reason for the improvement is evident: heavy use is made of averaging of the coefficients. Davidson [5] has recently published a most interesting stability condition which depends only on eigenvalue behavior. However, his method does not guarantee stability for any $a>0$ in the case of Example 1 and yields the rather restrictive sufficient conditions

$$
\frac{8}{9} \xi^{2}<1+f(t)<\frac{10}{9} \xi^{2}
$$

in the case of Example 2.

This last computation is clearly more in consonance with the viewpoint of imposing bounds on the variations of the time-varying parameters than on the averaging indicated by the quoted theorem. Along this vain, Brockett [6] has considered the system

$$
\ddot{x}+2 \dot{x}+f(t) x=0 \text {. }
$$


Brockett's stability results can be extended to the system of

Example 3 considered in the form

$$
\begin{aligned}
& \dot{x}=y-2 g(t) x \\
& \dot{y}=-[f(t)-2 \dot{g}(t)] x .
\end{aligned}
$$

It is easily seen, referring to Figure 1 and to the imposed bounds $0<\underline{g} \leqq g(t) \leqq \bar{g}, 0<\alpha \leqq f(t)-2 \dot{g}(t) \leqq \beta$, that the worst possible trajectories in the sense of stability that can be achieved are given by the autonomous equations

$$
\begin{aligned}
& \dot{x}=y-2 \underline{g x} \\
& \dot{y}=-\alpha x
\end{aligned}
$$

in region $I$ and in region II by

$$
\begin{aligned}
& \dot{x}=y-2 \underline{g x} \\
& \dot{y}=-\beta x
\end{aligned}
$$

and by extensions symmetric about the origin. Considering the trajectory $A B$ emerging from $(1,0)$ and the trajectory $E D$ passing through $(-1,0)$, it is clear that a sufficient condition for stability of the solutions is that

$$
x_{B} \leqq x_{E}
$$


and for asymptotic stability that the strict inequality hold. A simple but somewhat tedious computation of the solutions of these equations yields that the above condition becomes

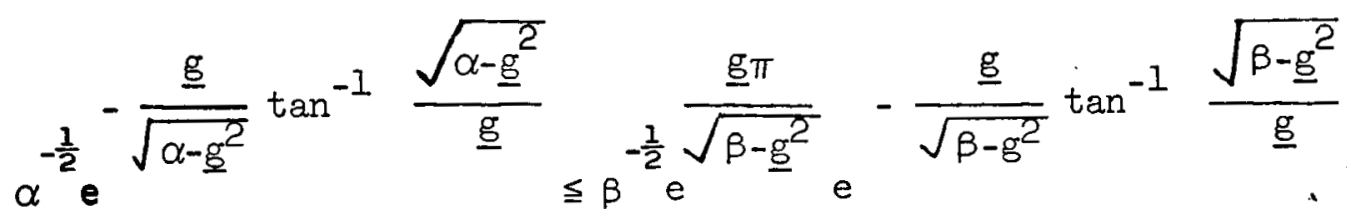

For $g(t) \equiv I$ this condition reduces to the one given by Brockett. This approach is similar to one followed by Aggarwal [7] for nonlinear second order systems. 
[1] Brockett, R. W. and I. J. Forys, "On the stability of systems containing a time-varying gain", Proceedings of 2nd Allerton Conference on Circuit and System Theory, 1964.

[2] Marcus, L., Yamabe, H., "Global stability criteria for differential systems", Osaka Math. J., vol. 12, pp. 305-317, 1960 .

[3] Infante, E.F., "On the stability of linear non-autonomous random systems", Journal of Applied Mechanics, vol. 36, no. 1, March 1968, p. 7 .

[4] Michael, G. J., "Explicit stability criteria for damped Mathieu equation", IEEE Transactions on Automatic Control, vol. AC-12, no. 3, June 1967.

[5] Davidson, E. J., "The stability of an $n^{\text {th }}$ order nonlinear timevarying differential system", IEEE Transactions on Automatic Control, vol. AC-13, no. 1, February 1968.

[6] Brockett, R. W., "Variational methods for stability of periodic equations, Differential Equations and Dynamical Systems", (Proceedings of the International Symposium held at the University of Puerto Rico, Mayaguez). Academic Press, 1967.

[7] Aggarwal, J. K., "Nonlinear oscillations: amplitide bounds for second-order systems", Journal of Franklin Institute, vol. 282, no. 1, July 1966. 


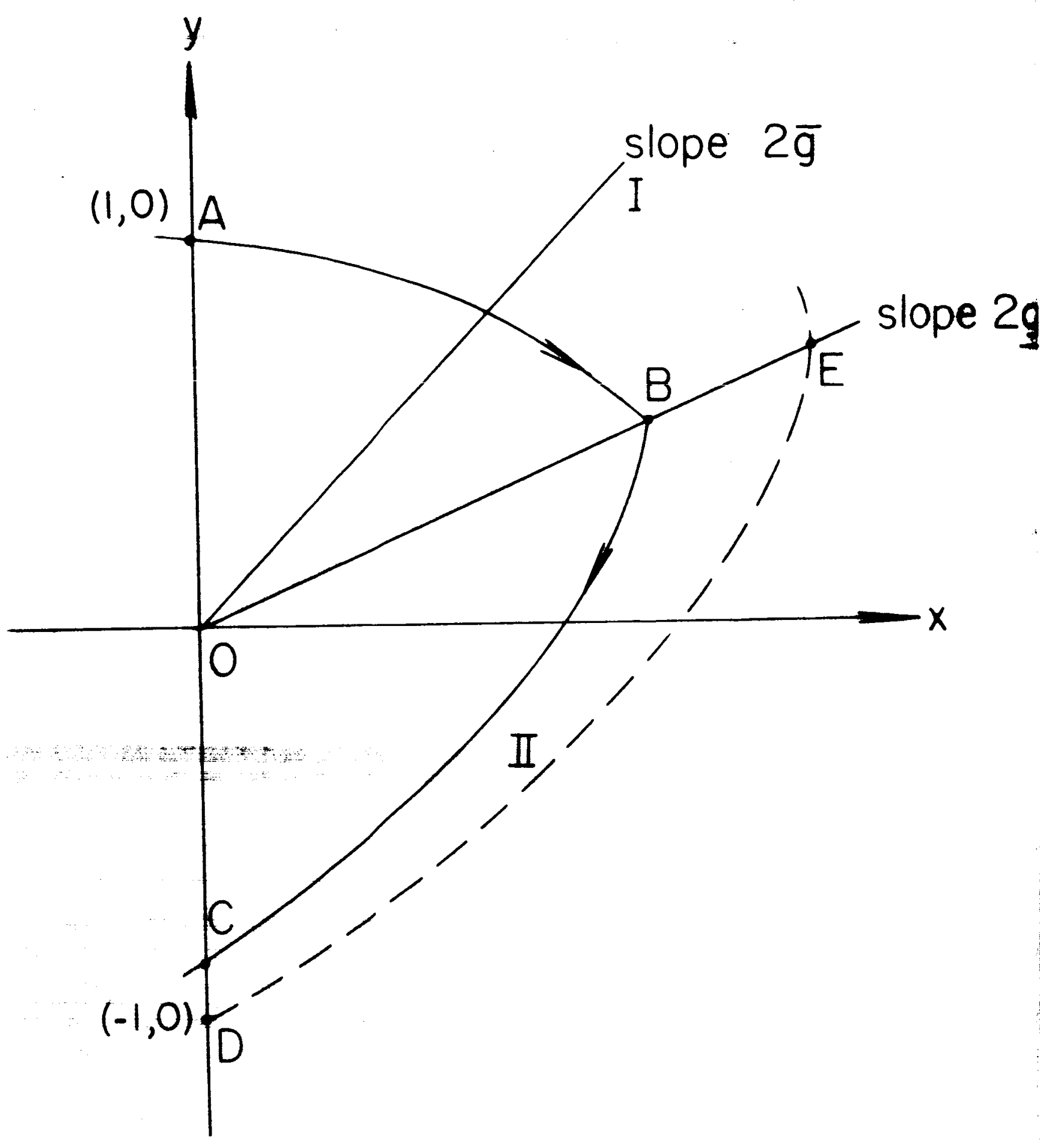

FIGURE I 